\title{
Pcf1, a large subunit of CAF-1, required for maintenance of checkpoint kinase Cds1 activity
}

\author{
Tatsuki Kunoh ${ }^{1}$ and Toshiyuki Habu²
}

\begin{abstract}
Highly conserved chromatin assembly factor 1 (CAF-1) is required for histone deposition onto newly synthesized DNA to maintain genome stability. This study shows that the fission yeast Pcf1, the large subunit in CAF-1, is crucial for maintaining checkpoint kinase Cds1. Chromatin recruitment of Cds1 is enhanced by Pcf1 overproduction but is attenuated by the $\Delta p c f 1$ mutation. Mutation of acetylation sites in the histone $\mathrm{H} 4$ tail abrogates the chromatin recruitment of Pcf1, which resembles that of Cds1 as reported previously. The present results provide evidence that chromatin recruitment of Pcf1, moderated by Clr6-HDAC activity, is essential for inactivating Cds1.
\end{abstract}

Keywords: Chromatin; Pcf/CAF-1; Cds1; Clr6-HDAC; Histone H4; Fission yeast

\section{Background}

In eukaryotes, DNA is assembled into a nucleoprotein complex called chromatin. The fundamental repeating unit of chromatin is the nucleosome in which DNA is wrapped around an octamer of core histone proteins including two $\mathrm{H} 2 \mathrm{~A} / \mathrm{H} 2 \mathrm{~B}$ dimers and an $(\mathrm{H} 3 / \mathrm{H} 4)_{2}$ tetramer (Ridgway and Almouzni 2000). The nucleosome is assembled primarily during DNA replication. Newly synthesized histones are deposited onto duplicated DNA along the replication fork with the aid of specialized assembly proteins (EhrenhoferMurray 2004). Nucleosome assembly is also required for DNA repair and transcription. After nucleotide excision repair or double-strand break repair, the nucleosome is reassembled at the site of repaired DNA (Linger and Tyler 2005; Lewis et al. 2005; Nabatiyan et al. 2006). Transcription is generally thought to correlate with a reduction of histone density on the DNA template allowing for the passage of the transcription machinery. It was reported that nucleosomes were selectively removed and replaced by chromatin remodeling factors and histone chaperones $(\mathrm{Li}$ et al. 2007; Saunders et al. 2006; Ehrenhofer-Murray 2004).

The conserved CAF-1 (chromatin assembly factor-1) protein complex was first identified in human cells by its ability to stimulate nucleosome assembly in vitro (Smith and Stillman 1989). This protein complex, which is associated with a DNA-sliding clump protein PCNA (Proliferating

\footnotetext{
* Correspondence: habu.toshiyuki.6x@kyoto-u.ac.jp

${ }^{2}$ Radiation Biology Center, Kyoto University, Kyoto 606-8501, Japan

Full list of author information is available at the end of the article
}

Cell Nuclear Antigen), localizes in the replication fork and deposits a newly synthesized acetylated form of histones H3 and H4 (Shibahara and Stillman 1999). Once assembled in nucleosomes, the histones are promptly deacetylated by histone deacetylases (HDACs). However, both CAF-1 and acetylated-H4 are transiently maintained at the late replication foci, suggesting that CAF-1 and HDACs might interact during chromatin maturation (Taddei et al. 1999). Indeed, CAF-1 plays an essential role in maintaining constitutive heterochromatin in yeast (Huang et al. 2007). Despite the established role of CAF-1 in replication-coupled nucleosome assembly, deletion of any of the three CAF-1 genes has minimal adverse effect on normal growth in yeast (Kaufman et al. 1997), suggesting that other histone chaperones such as Asf1 (anti-silencing factor 1) and HIR/HIRA (histone regulation) may function in $\mathrm{H} 3 / \mathrm{H} 4$ assembly cooperatively with CAF-1 (Tamburini et al. 2006; Greenall et al. 2006).

The DNA replication checkpoint has a surveillance function that regulates origin firing, maintains the integrity of the stalled replication fork, and prevents cells from proceeding to mitosis before completion of the DNA replication (McNeely et al. 2013). The replication checkpoint pathway is highly conserved in eukaryotes. In mammalian cells an initial defect is sensed by a protein kinase, termed ATR, which transmits signals to Chk2 effector kinase. In fission yeast, the replication checkpoint requires the ATR ortholog Rad3 and Chk2 ortholog Cds1 (McGowan and Russell 2004). In budding

\section{Springer}

(c) 2014 Kunoh and Habu; licensee Springer. This is an Open Access article distributed under the terms of the Creative Commons Attribution License (http://creativecommons.org/licenses/by/2.0), which permits unrestricted use, distribution, and reproduction in any medium, provided the original work is properly cited. 
yeast, the checkpoint effector kinase Rad53 directly interacts with Asf1 and regulates chromatin assembly to promote cell survival against DNA damage and replication block (Sharp et al. 2005). Although little is known about the mechanism, CAF-1 is associated with the full activation of the Chk1-dependent checkpoint pathway upon a replication stress in vertebrate cells (Takami et al. 2007). These reports indicate the importance of histone assembly in the S-phase checkpoint response. In budding yeast, hyperacetylation of H3K56, a hallmark of replication-associated lesions, results in activation of Rad53 (Maas et al. 2006). Deacetylation of H4 tail is required for inactivation of Cds1 upon replication stress in fission yeast (Kunoh et al. 2008), suggesting that the acetylation status of histones could affect the checkpoint response. However, how the acetylation status affects histone assembly and thereby checkpoint maintenance in response to the replication block remains unsolved.

In the present paper, we show that Pcf1, the large subunit of fission yeast CAF-1, is required for chromatin organization, maintenance of Cds1 activity, and its chromatin recruitment. Further, chromatin recruitment of Pcf1 depends on the acetylation status of the H4 tail regulated by the Clr6-HDAC, so that it may contribute to the checkpoint inactivation after replication stress.

\section{Results}

Pcf1, the large subunit of CAF-1, is involved in chromatin organization and interacts genetically with the replication checkpoint pathway component Cds1

During DNA replication, histone deposition is critical for chromatin organization. Among histone chaperones, CAF-1 is considered to be responsible for this process in vertebrate cells (Taddei et al. 1999). In fission yeast cells, proteins homologous to the CAF-1 subunits were shown to form a complex that associates with PCNA (Dohke et al. 2008). Nevertheless, whether CAF-1 is required for chromatin organization in fission yeast remains unclear. To answer this query, we isolated bulk chromatin from wild type and $\Delta p c f 1$, the mutant lacking $p c f 1^{+}$gene encoding the large subunit of CAF-1 (Dohke et al. 2008), then digested the chromatin with micrococcal nuclease (MNase). As illustrated in Figure 1A, nucleosomecorresponding DNA fragments with fewer base pairs appeared earlier from the digested chromatin of $\Delta p c f 1$ mutant than that of the wild type. By 2 min after digestion, DNA fragments had already appeared in $\Delta p c f 1 \mathrm{mu}-$ tant but not in the wild type. The intensity of the bands corresponding to the oligo-nucleosomes was stronger in the wild type than in the $\Delta p c f 1$ mutant at 20 and 60 min after digestion. This earlier digestion of bulk chromatin

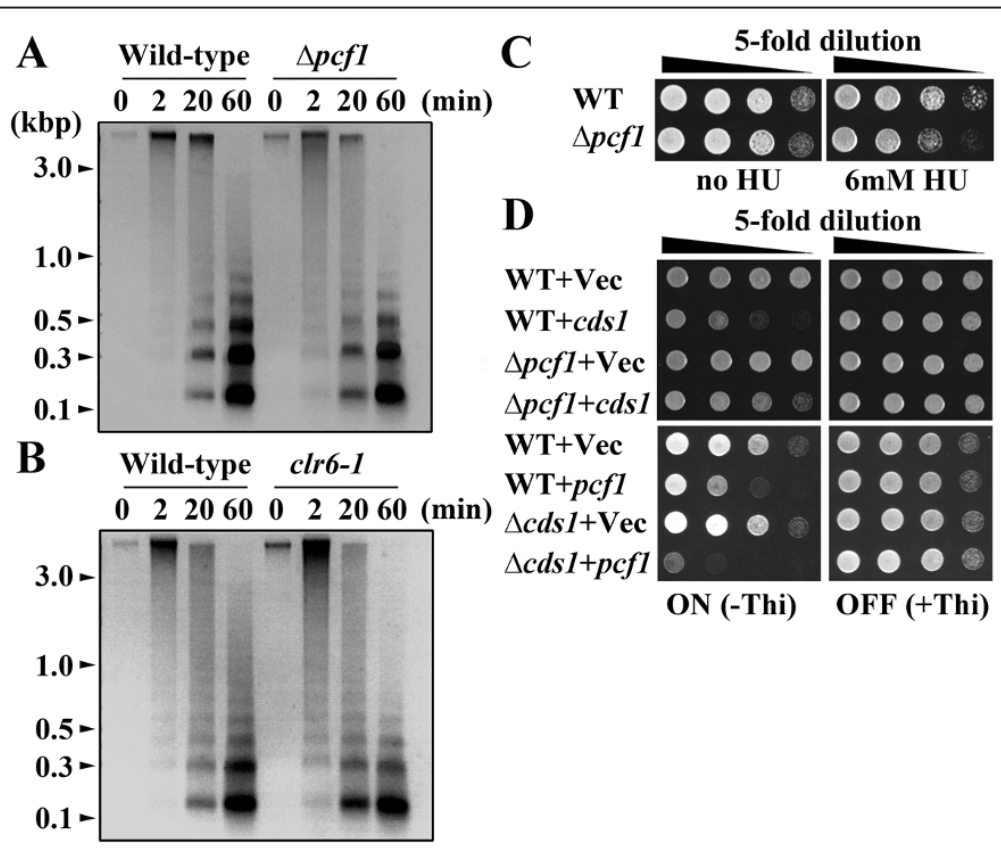

Figure 1 Electrophoresis patterns of MNase-digested DNA of $\triangle p c f 1$ mutant and wild type cells, their sensitivity to hydroxyurea (HU) and genetic interactions between $\mathbf{c d s} \mathbf{1}^{+}$and $\boldsymbol{p} \mathbf{c f} \mathbf{1}^{+}$. (A, B) Agarose-gel electrophoresis patterns of DNA prepared from enzyme-digested chromatin of wild type and $\Delta p c f 1$ mutant (A) and clr6-1 mutant (B) cells. Note the early appearance of smaller DNA fragments, corresponding to nucleosomes, from digested chromatin of both $\Delta p c f 1$ and clr6-1 mutants relative to that of the wild type. (C) Sensitivity of wild type and $\Delta p c f 1$ mutant cells to $\mathrm{HU}$. Both cells grew similarly on the plates without $\mathrm{HU}$ regardless of its dilution, whereas $\Delta p c f 1$ mutant cells grew slowly relative to wild type cells on plates amended with $6 \mathrm{mM} \mathrm{HU}$. (D) Growth of wild type, $\Delta p c f 1$ and $\Delta c d s 1$ mutant cells harboring the empty vector (Vec), pREP-cds $1^{+}$, or pREP-pcf1 ${ }^{+}$in the presence or absence of thiamine. For a detailed comparison of the growth of these cells, see the text. 
in the $\Delta p c f 1$ mutant was confirmed in repeated experiments. As a positive control, clr6-1 mutant was subjected to MNase digestion, since it was hypothesized that Clr6-HDAC participated in global deacetylation of histones, affecting chromatin maturation throughout the genome (Grewal 2000). The pattern of small fragment DNAs digested by MNase in clr6-1 mutant was quite similar to that in the $\Delta p c f 1$ mutant (Figure $1 \mathrm{~A}, \mathrm{~B}$ ). Thus, the bulk chromatin from $\Delta p c f 1$ and clr6-1 mutant cells is likely to be more sensitive to MNase relative to that from the wild type cells. The results suggest that the global chromatins may readily relax in the $\Delta p c f 1$ mutant cells, although such a conclusion cannot be drawn until the band intensities of Figure 1A are quantified.

Because deletion of $p c f 1^{+}$did not affect normal growth of yeast cells (Figure 1C, left), CAF-1 including Pcf1 might function cooperatively in chromatin assembly with other histone chaperones such as Asf1 and HIR/ HIRA proteins as already mentioned. On the other hand, the $\Delta p c f 1$ mutant cells grew slowly relative to the wild type cells under the DNA replication stress imposed by hydroxyurea (HU) (Figure $1 \mathrm{C}$, right), suggesting that Pcf1 might be involved in maintenance of genome stability during the slowed DNA replication in fission yeast.

To determine whether Pcf1 is involved in the DNA replication checkpoint pathway, we examined the genetic interaction of Pcf1 with an effector kinase Cds1. Consistent with a previous report (Boddy et al. 1998), overexpression of $c d s 1^{+}$led to slow growth of wild type cells, when the nmt1 promoter was activated under a thiamine-free condition (WT + Vec vs. WT + cds1 in the upper left of Figure 1D). However, in the thiamine-free condition, cell growth notably improved with deletion of $p c f 1^{+}$(WT $+c d s 1$ vs. $\left.\Delta p c f 1+c d s 1\right)$, although it was slower than that of the vector control $(\Delta p c f 1+\mathrm{Vec}$ vs. $\Delta p c f 1+c d s 1)$. On the other hand, the slow growth enforced by overexpression of $p c f 1^{+}$in wild type cells (WT + Vec vs. WT + pcf1 in the lower left of Figure 1D) was markedly enhanced by deletion of $c d s 1^{+}(\mathrm{WT}+p c f 1$ vs. $\Delta c d s 1+p c f 1)$. By contrast, in the presence of thiamine, growth of these cells was not affected (Figure 1D, upper and lower right), since $c d s 1^{+}$or $p c f 1^{+}$driven by $n m t 1$ promoter was expressed at low level. Apparently, a strong genetic correlation exists between $p c f 1^{+}$and $c d s 1^{+}$. These results directed us to examine whether Pcf1 was involved in the DNA replication checkpoint response.

\section{Pcf1 required for maintenance of Cds1 activity}

Tanaka et al. (2001) reported that the phosphorylated Cds1 migrated slowly on SDS-PAGE when the DNA replication checkpoint was activated. In the present study, therefore, this mobility shift was used as a marker to examine how the lack of Pcf1 affected the DNA replication checkpoint response. The mobility pattern of the cell extracts from the wild type and $\Delta p c f 1$ (Figure 2A) showed that phosphorylation of Cds1 in the former cells was sustained for 2-6 h after the HU treatment, whereas for only $2-4 \mathrm{~h}$ in the latter cells. These results obviously show that Pcf1 plays a key role in keeping the active state of Cds1. The septation index (Figure 2B), micrographs (Figure 2C) and count data (Figure 2D) jointly show that the $\Delta p c f 1$ mutant cells proceeded to $\mathrm{G} 2 / \mathrm{mi}$ tosis earlier than the wild type cells did in the presence of HU, suggesting that Pcf1 might be crucial for checkpoint maintenance upon replication stress.

Cds1 is recruited to chromatin in a Pcf1-dependent manner Binding stability of Cds1 to chromatin was examined by Triton extraction and immunofluorescence as reported previously (Kunoh et al. 2008). This technique is able to detect the chromatin-bound Cds1 in the cells after the Triton extraction. We previously reported that Cds1 was removed during Triton extraction of asynchronously cultured cells but not from cells in which the replication checkpoint was activated by $\mathrm{HU}$ because Cds1 remained bound to chromatin (Kunoh et al. 2008). As expected, immunofluorescence images of the wild type and $\Delta p c f 1$ cells $6 \mathrm{~h}$ after $\mathrm{HU}$ treatment (Figure 3A) illustrated that Cds1 was tightly bound to DAPI-stained chromatin in the wild-type (chromatin binding affinity of Cds1 = 83\%) but not in the $\Delta p c f 1$ mutant (19\%), which is consistent with the aforementioned evidence that Cds1 was still activated $6 \mathrm{~h}$ after HU treatment in wild type cells but not in $\Delta p c f 1$ mutant cells as shown in Figure 2A. Because these results support the conclusion that Pcf1 is required to maintain Cds1 activity, we assumed that overexpression of $p c f 1^{+}$could activate Cds1. However, overexpression of $p c f 1^{+}$did not cause the mobility shift in the absence of thiamine (Figure 3B). Nevertheless, it resulted in tight binding of Cds1 to chromatin even in the absence of HU (Figure $3 \mathrm{C}$ and D), indicating that overexpression of $p c f 1^{+}$does not cause phosphorylation of Cds1 but rather recruitment of Cds1 to chromatin.

Figure three B of Kunoh et al. (2008) of an immunoblot using the tetra-Ac histone $\mathrm{H} 4$ antibody illustrated that acetylation of $\mathrm{H} 4$ in the wild type was abrogated after HU treatment and that, in the same blot, the band intensity of the histone H4.2 K8A K16G mutant was weak, similar to the wild type after HU treatment, whereas the latter was higher than the former when cells were cultured asynchronously. In contrast, acetylation of H4 was not abrogated in clr6-1 mutant. These results led us to consider that at least acetylation at $\mathrm{K} 8$ and/or K16 residues of $\mathrm{H} 4$ tail, removed by Clr6-HDAC, was associated with response to HU treatment. Furthermore, phosphorylation and chromatin recruitment of Cds1 in the clr6-1 mutant were sustainably maintained once activated. Conversely, the H4.2 K8A K16G mutant failed to 


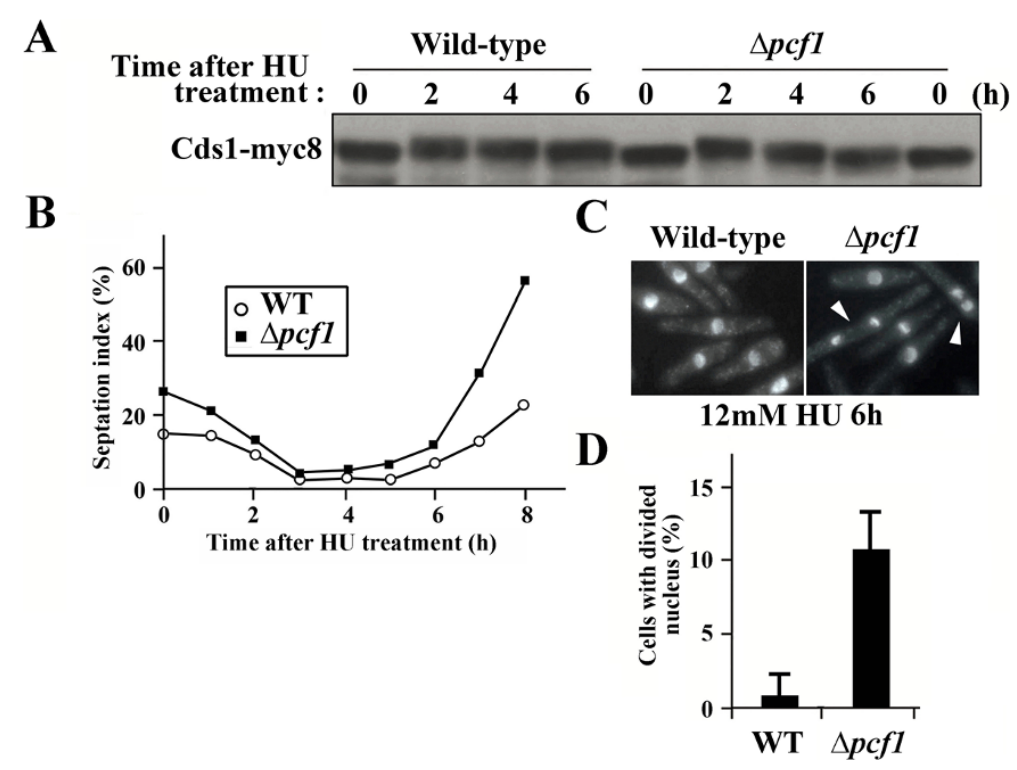

Figure 2 Disturbance of the activation of the DNA replication checkpoint by deletion of $p c f 1^{+}$. (A) Mobility of Cds1 prepared from wild type and $\Delta p c f 1$ mutant on SDS-PAGE at various times after HU treatment. (B) Time course of septum formation in cells (septation index) in $\Delta p c f 1$ mutant (closed square) and wild type cells (open circle) after HU treatment. (C) DAPI-stained cells of wild type and $\Delta p c f 1$ mutant $6 \mathrm{~h}$ after HU treatment. Arrowheads indicate cells with a segregated nucleus. (D) Incidence of cells with a segregated nucleus $6 \mathrm{~h}$ after HU treatment.

keep the checkpoint active due to extraordinary phosphorylation and reduction in the recruitment of Cds1 to chromatin upon replication stress (Kunoh et al. 2008), which resembles the situation in the $\Delta p c f 1$ mutant (Figures $2 \mathrm{~A}$ and $3 \mathrm{~A}$ ).

\section{Pcf1 recruitment to chromatin is regulated by the acetylation state of $\mathrm{H} 4$}

We next tested whether Clr6-HDAC and Pcf1 functioned to regulate Cds1 in the same pathway. Disruption of $p c f 1^{+}$made the $c l r 6-1$ mutant cells extremely sensitive to $\mathrm{HU}$, similar to the case in the $\Delta c d s 1$ clr6-1-double mutant (Figure 4A, left). In addition, on the basis of the septation index (Figure 4B), the deletion of $p c f 1^{+}$directed the clr6-1 mutant cells toward G2/mitosis upon replication stress, similar to the deletion of $c d s 1^{+}$(Kunoh et al. 2008). It is evident that the slow growth resulting from $p c f 1^{+}$overexpression was partially suppressed by the H4.2 K8A K16G mutation (WT + pcf1 vs. K8A $\mathrm{K} 16 \mathrm{G}+p c f 1$ in Figure 4C), when compared with growth of $\mathrm{WT}+\mathrm{Vec}$ vs. WT $+p c f 1$ in Figure $4 \mathrm{C}$. Results suggest that deacetylation of $\mathrm{H} 4$ by Clr6-HDAC can regulate function of Pcf1.

Deacetylation of $\mathrm{H} 4$ in HU-treated $\Delta p c f 1$ mutant cells was comparable to that in the similarly treated wild-type cells (Figure 4D), suggesting that Clr6-HDAC activation could proceed adequately in the absence of Pcf1. This assumption led us to examine whether Clr6-HDAC activation was required for removal of Pcf1 from the chromatin. Figure 4E, showing that $\alpha$-tubulin and histone H3 were detected only in the chromatin-unbound and -bound fractions, respectively, proved the proper performance of the assay. Recruitment of Pcf1-GFP to chromatin upon replication stress was abrogated by H4.2 K8A K16G mutation, which is clear in the comparison of the chromatinbound fractions of wild type and H4.2 K8A K16G mutant cells at $4 \mathrm{~h}$ in Figure 4E. Results led us to consider that removal of Pcf1 from chromatin in response to Clr6HDAC-dependent H4-deacetylation might be sufficient to inactivate Cds1 upon replication stress. In another way, it is also considerable that Cds1 is recruited to remove Pcf1 from chromatin. Experiments are ongoing to test these possibilities and examine whether chromatin binds to Pcf1 when $c l r 6^{+}$is mutated and/or overexpressed.

\section{Discussion}

Modification of chromatin structure caused by mutation of $p c f 1^{+}$(Figure 1A) and enhanced sensitivity of $\Delta p c f 1$ to HU (Figure 1C) suggest that Pcf1 could be required for replication-coupled nucleosome assembly in fission yeast. Genetic and biological analyses revealed that Pcf1 was crucial for sustained activity and chromatin recruitment of Cds1 upon replication stress (Figures 1D, 2, and $3 \mathrm{~A})$. In addition, overexpression of $p c f 1^{+}$led to the recruitment of Cds1 to chromatin (Figure 3C and D) without the spontaneous phosphorylation of Cds1 (Figure 3B). Thus, we emphasize here that Pcf1 could be critical in the recruitment of Cds1 to chromatin and that phosphorylation of Cds1, which is regulated through the conventional checkpoint system, could not be required for this process, 


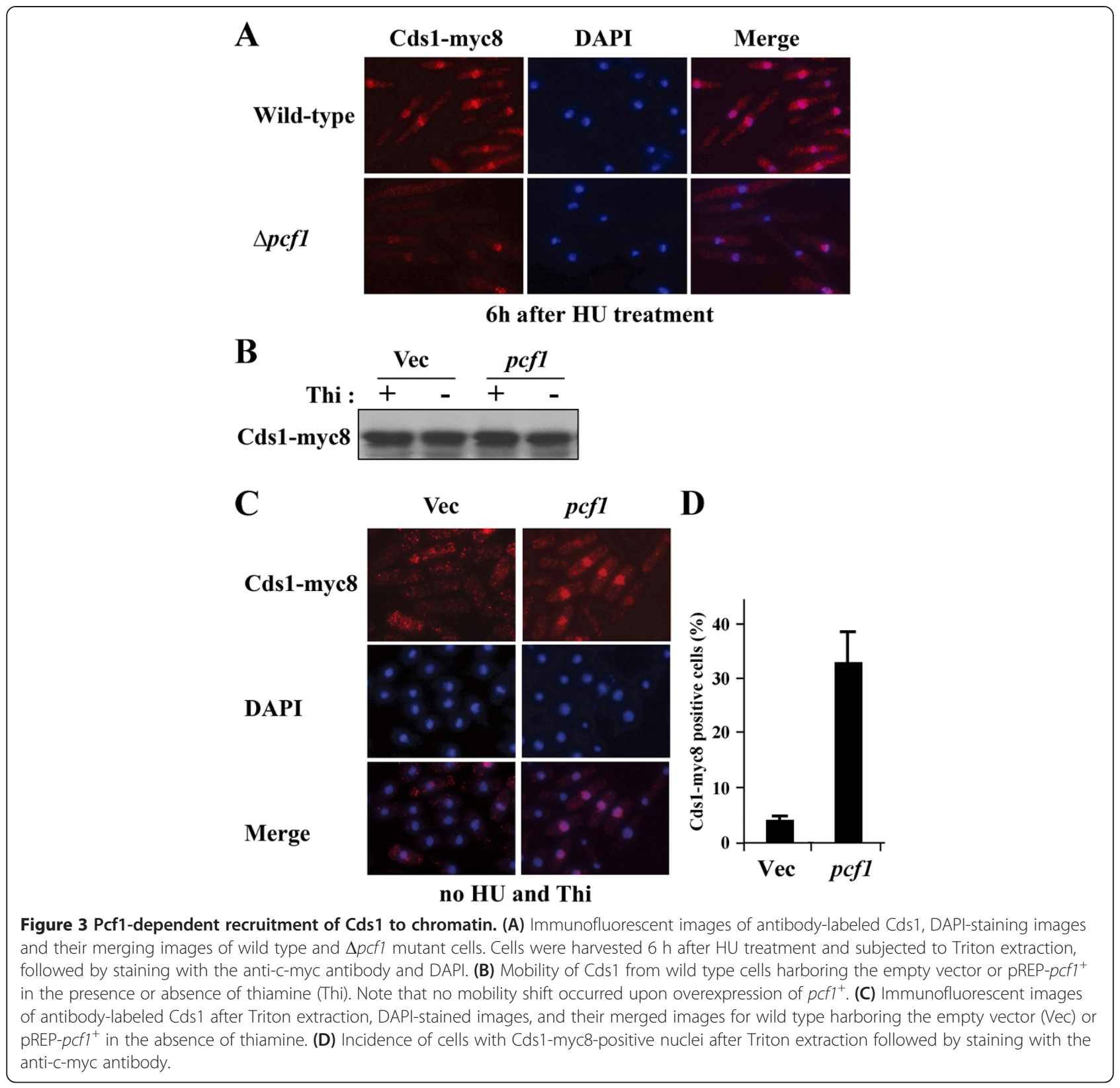

although it has been generally accepted that Cds1 phosphorylation is an essential key for activating the DNA replication checkpoint (Tanaka et al. 2001). Although Cds1 remains in an active state even after DNA replication is completed in the clr6-HDAC mutant (Kunoh et al. 2008), the $\Delta p c f 1$ mutation directed the clr6-HDAC mutant cells toward mitosis (Figure 4B), suggesting that H4-acetylationdependent maintenance of checkpoint could be regulated by Pcf1. Slow cell growth caused by overexpression of $p c f 1^{+}$was suppressed by mutation of the $\mathrm{H} 4$ acetylation sites (Figure 4C). In addition, recruitment of Pcf1 to chromatin was lost with this mutation (Figure 4E). These results indicate the possibility that Pcf1 is regulated by $\mathrm{H} 4$ acetylation. Taken together all these data, it is inferred that deacetylation of $\mathrm{H} 4$ by Clr6-HDAC could regulate Cds1 via Pcf1. As mentioned, Haldar and Kamakaka (2008) reported that fission yeast cells deficient in Hst4-HDAC, which is responsible for $\mathrm{H} 3-\mathrm{K} 56$ deacetylation, were sensitive to DNA-damaging agents. Consistently, an effector kinase of DNA damage checkpoint, Chk1, was activated constitutively in the Hst4-HDAC-deficient cells (Haldar and Kamakaka 2008). These results and our current findings suggest that discontinuation of histone acetylation might generate signals to complete histone assembly on damaged or slowly replicating DNA so as to inactivate the S-phase checkpoint pathways. 

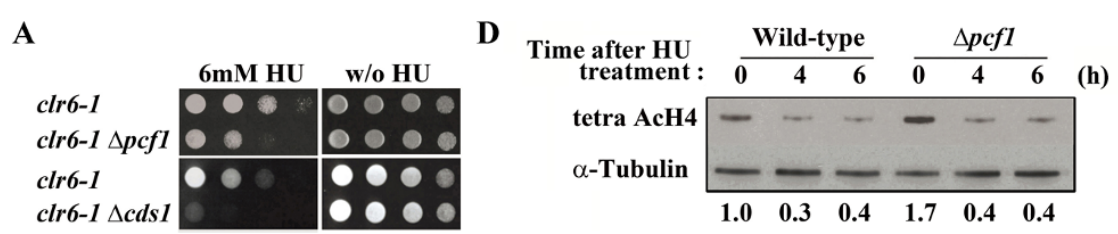

B

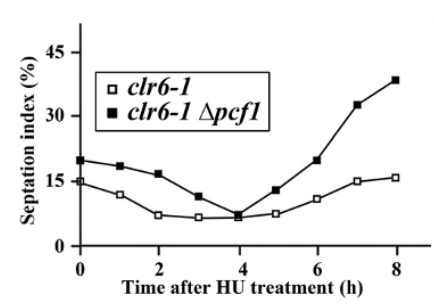

$\mathbf{E}$

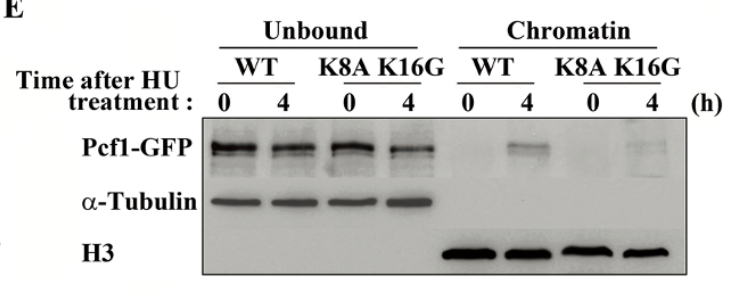

\begin{tabular}{|c|c|c|}
\hline C & - Thi & + Thi \\
\hline WT + pREP2 & 000 策 & 0000 \\
\hline $\mathrm{WT}+$ pREP2-pcfl & 003 & 0000 \\
\hline K8A K16G + pREP2 & 000 & 0000 \\
\hline K8A K16G + pREP2-pcf1 & $00 \%$ & 000 \\
\hline
\end{tabular}

Figure 4 Recruitment of Pcf1 to chromatin regulated by the acetylation state of H4. (A) Cell growth of clr6-1 single mutant and clr6-1 $\Delta p c f 1$ and clr6-1 $\Delta c d s 1$ double mutant cells in the presence or absence of $\mathrm{HU}$. Note the slower growth of the double mutants relative to that of clr6-1 single mutant in the presence of HU. (B) Septation index of clr6-1 single (open square) and clr6-1 $\Delta p c f 1$ double mutant (closed square) at various times after the $\mathrm{HU}$ treatment. (C) Cell growth of wild type and H4.2 K8A K16G mutant cells harboring the empty vector or pREP1-pcf1 ${ }^{+}$in the presence or absence of thiamine. Note that slow growth by Pcf1 overproduction in wild type cells was suppressed by H4 K8A K16G mutation. (D) Immunoblotting of acetylated $\mathrm{H} 4$ (tetra $\mathrm{AcH} 4$ ) from the wild-type and $\mathrm{\Delta pcf1}$ mutant cells at various times after the HU treatment. As a loading control, an anti-a-tubulin antibody was used. (E) Immunoblotting of chromatin-bound and -unbound fractions prepared from cell extracts of wild-type and H4.2 K8A K16G mutant cells at indicated times after HU treatment.

RBAp48 that associates with the complexes including CAF-1, HDAC, and NURF in a broad range of species (Marheineke and Krude 1998; Nakayama et al. 2003) is thought to act as a liaison factor among these complexes. Therefore, RBAp48 could be one of the best candidates to regulate histone deposition and deacetylation, although it remains unclear whether RBAp48 is also required to maintain the DNA replication checkpoint. We conclude that nucleosome assembly by CAF-1, including Pcf1 and Clr6-HDAC, could be crucial for inactivating Cds1 after replication stress.

\section{Summary}

In this study, we found that the deletion mutant of the $p c \mathrm{fl}^{+}$gene, which encodes the large subunit of fission yeast CAF-1 failed to maintain the activity of Cds1, an effector kinase of DNA replication checkpoint. Consistently, chromatin recruitment of Cds1 in response to replication stress was also abolished by deletion of $p c 1^{+}$. These results suggest the conserved function of CAF-1 could contribute to maintain checkpoint machineries in an active state. Intriguingly, Pcf1 overproduction enhanced the chromatin recruitment of Cds1 even in the absence of replication stress. In addition, the growth inhibition induced by the overproduction of Cds1 was significantly suppressed by the deletion of $p c f 1^{+}$, being consistent with the notion that CAF-1 might be crucial for sustained activation of the checkpoint effector Cds1. Importantly, chromatin recruitment of Pcf1 was negated by the mutation at the acetylation sites in $\mathrm{H} 4$ after replication stress. Furthermore, growth inhibition induced by Pcf1 overproduction was partially suppressed by $\mathrm{H} 4$ mutation. On the basis of these results, we propose that $\mathrm{H} 4$ deacetylation by Clr6-HDAC could modulate Cds1 activity by regulating recruitment of Pcf1 to chromatin.

\section{Materials and methods}

Strains, plasmids and media

The strains used in this study are the derivatives of Schizosaccharomyces pombe $h^{-} 972$ and $h^{+} 975$ (Beach et al. 1985). The mutant, clr6-1, and histone H4 mutant, H4.2 K8A K16G, were also used (Bjerling et al. 2002; Mellone et al. 2003). Standard fission yeast techniques and media were employed (Moreno et al. 1991). To construct pREP- $c d s 1^{+}$and pREP- $p c f 1^{+}$, the ORF of $c d s 1^{+}$and $p c f 1^{+}$ genes were independently amplified by PCR and cloned into pREP2 (provided by National BioResource Project, Japan). To repress transcription from the $n m t 1$ promoter of pREP2, the cells were grown in the EMM2 minimal medium supplemented with $4 \mu \mathrm{M}$ thiamine. For examining hydroxyurea $(\mathrm{HU})$ sensitivity of the cells, $12 \mathrm{mM}$ $\mathrm{HU}$ was added to the medium. For spot assays, exponentially growing cultures were serially diluted five-fold, and 
each dilution was spotted onto agar plates containing $\mathrm{HU}$ and/or thiamine.

\section{Gene disruption and tagging}

Gene disruption was performed as previously reported (Rothstein 1983). For myc8 or GFP tagging, an 1-kbp fragment of the C-terminus region of $c d s 1^{+}$and $p c f 1^{+}$ genes were inserted into pYC11-myc8 and pYC116xGFP, respectively, and the resultant plasmids were introduced into both wild-type and mutant strains. Gene disruption and gene replacement were confirmed by southern blotting and immunoblotting.

\section{Cell extracts and immunoblot}

For examining mobility of Cds1 and acetylated $\mathrm{H} 4$ on SDS-PAGE, exponentially growing cells of the wild-type and the $\Delta p c f 1$ mutant were treated with $6 \mathrm{mM} \mathrm{HU}$, then a cell extract was prepared from 0 to $6 \mathrm{~h}$ according to previous methods (Kunoh et al. 2008). For immunoblots, anti-c-myc (1:7500; Covance Inc., Princeton, NJ), antitetra acetylated histone H4 (1:5000; Bio-Rad AbD Serotec Ltd., Oxfordshire, UK) (described as tetra AcH4 in Figure 4D), anti-Tat1 (1:5000; generously provided by Dr. A. Baines), anti-GFP (1:5000; Roche Applied Science, Mannheim, Germany), anti-H3 (1:2500; Merk, Darmstadt, Germany) (described as tetra H3 in Figure 4E) antibodies served as the primary antibodies. Goat HRP conjugatedanti-mouse (1:25000; Life Technologies, Carlsbad, CA) and anti-rabbit antibody (1:25000; Life Technologies) were used as the secondary antibodies.

\section{Triton extraction and immunofluorescence microscopy}

To monitor binding affinity of Cds1 to chromatin, Cds1myc8 was extracted with TritonX-100 and immunostained as described previously (Zhao et al. 2003; Kunoh et al. 2008). Counting of cells with either segregated or Cds1-myc8-positive nuclei was replicated at three times (50 cells per replicate) in the field of view at x1000; mean values with SD are given in Figures 2D and 3D, respectively. The chromatin binding affinity of Cds1 was evaluated as the number of Cds1-myc8-positive nuclei per total number of DAPI-stained nuclei.

\section{MNase digestion of the bulk chromatin}

The status of the global chromatins in $\Delta p c f 1$ and clr6-1 mutant was monitored using MNase (micrococcal nuclease) digestion assay. Isolation of bulk chromatin and MNase digestion were performed as described previously (Takahashi et al. 1992).

\section{Chromatin fractionation assay}

To examine whether recruitment of Pcf1 to chromatin was affected by $\mathrm{H} 4$ acetylation, we first treated wild type and $\Delta p c f 1$ mutant cells with $12 \mathrm{mM} \mathrm{HU}$ at designated times before chromatin fractionation. The fractionation procedure was generously provided by Drs. M. Sadaie and J. Nakayama (RIKEN, Kobe, Japan). Approximately $2.5 \times 10^{8}$ cells of wild type or $\Delta p c f 1$ mutant were harvested and washed in ice-cold STOP buffer $(150 \mathrm{mM}$ $\mathrm{NaCl}, 50 \mathrm{mM} \mathrm{NaF}, 10 \mathrm{mM}$ ethylenediaminetetraacetic acid, and $1 \mathrm{mM} \mathrm{NaN}$ ). Cells were resuspended in $1 \mathrm{~mL}$ of PEMS buffer (100 mM PIPES, $1 \mathrm{mM}$ EGTA, $1 \mathrm{mM}$ $\mathrm{MgCl}_{2}, 1.2 \mathrm{M}$ sorbitol, pH6.9) containing $1 \mathrm{mg} / \mathrm{mL}$ each of lysing enzyme (Sigma, St. Louis, MO) and zymolyase $100 \mathrm{~T}$ (Seikagaku Co., Tokyo, Japan), followed by incubation at $37^{\circ} \mathrm{C}$ for $20 \mathrm{~min}$. After washing out lysing enzyme and zymolyase $100 \mathrm{~T}$ with $1.2 \mathrm{M}$ sorbitol, we then suspended the spheroplasts in HBS-T buffer $(25 \mathrm{mM}$ MOPS, $60 \mathrm{mM} \beta$-glycerophosphate, $15 \mathrm{mM} \mathrm{MgCl}_{2}$, $15 \mathrm{mM}$ EGTA, $1 \mathrm{mM}$ dithiothreitol, $0.2 \mathrm{mM} \mathrm{Na}_{3} \mathrm{VO}_{4}$, $1 \mathrm{mM}$ phenylmethylsulfonyl fluoride, $1.2 \mathrm{M}$ sorbitol, and $0.5 \%$ Triton X-100, pH7.2) and incubated on ice for $5 \mathrm{~min}$. After centrifugation at $13,000 \mathrm{rpm}$ for $20 \mathrm{~min}$, the supernatant was saved as an unbound fraction. Then pellet was washed twice in HBS-T buffer, resuspended in the initial volume of HBS-T buffer, and saved as a chromatin fraction. Both fractions were subjected to immunoblots as described above. Proper performance of the assay was confirmed by using anti- $\alpha$-tubulin and antiH3 antibodies as loading controls. Fractionated Pcf1GFP was detected by using the anti-GFP antibody.

\section{Septation index}

In fission yeast, septum formation is one indicator of the completion of mitosis. Thus, we counted the number of cells with a septum in the field of view of a light microscope at x1000 to determine a septum index. The counts were replicated at least three times (50 cells per replicate).

\section{Abbreviations \\ CAF-1: Chromatin assembly factor-1; HDAC: Histone deacetylase; ATR: ATM and Rad3 related; Chk: Checkpoint kinase; Cds1: checking DNA synthesis; Rad: radiation sensitive; RBAp48: Rb-associated protein $\mathrm{p} 48$; \\ NURF: Nucleosome Remodeling Factor; GFP: Green Fluorescent Protein; H3K56: Histone H3 Lysine 56; MNase: Micrococcal nuclease; HU: Hydroxyurea; WT: Wild type; Vec: Vector; Thi: Thiamine; DAPI: 4',6-Diamidino-2- phenylindole; EGTA: 2-aminoethyl ether tetraacetic acid; H4.2 K8A K16G: Histone H4.2 Lysine 8 to Alanine and Lysine 16 to Glycine.}

\section{Competing interests}

The authors declare that they have no competing interests.

\section{Authors' contributions}

TK designed the study, carried out the molecular and cellular studies, and drafted the manuscript. TH coordinated the study, and helped to draft the manuscript. Both authors read and approved the final manuscript.

\section{Acknowledgements}

We are particularly grateful to Drs. T. Matsumoto, M. Yanagida, K. Ekwall and R. C. Allshire for plasmids and strains. We thank Drs. K. Tanaka, J. Nakayama, M. Sadaie, T. Miyoshi and H. Kunoh for helpful discussion and procedures. We also acknowledge Dr. B. E. Hazen for reviewing and editing the manuscript. This work was supported by grants from the Ministry of Education, Culture, Sports, Science and Technology of Japan to TH. 


\section{Author details}

${ }^{1}$ Laboratory of Molecular Pharmacogenomics, School of Pharmaceutical Sciences, Kinki University, Higashiosaka 577-8502, Japan. ${ }^{2}$ Radiation Biology Center, Kyoto University, Kyoto 606-8501, Japan.

Received: 12 October 2013 Accepted: 14 January 2014 Published: 17 January 2014

\section{References}

Beach D, Rodgers L, Gould J (1985) ran $1^{+}$controls the transition from mitotic division to meiosis in fission yeast. Curr Genet 10:297-311

Bjerling P, Silverstein RA, Thon G, Caudy A, Grewal S, Ekwall K (2002) Functional Divergence between histone deacetylases in fission yeast by distinct cellular localization and in vivo specificity. Mol Cell Biol 22:2170-2181

Boddy MN, Furnari B, Mondesert O, Russell P (1998) Replication checkpoint enforced by kinases Cds1 and Chk1. Science 280:909-912

Dohke K, Miyazaki S, Tanaka K, Urano T, Grewal SI, Murakami Y (2008) Fission yeast chromatin assembly factor 1 assists in the replication-coupled maintenance of heterochromatin. Genes Cells 13:1027-1043

Ehrenhofer-Murray AE (2004) Chromatin dynamics at DNA replication, transcription and repair. Eur J Biochem 271:2335-2349

Greenall A, Williams ES, Martin KA, Palmer JM, Gray J, Liu C, Whitehall SK (2006) Hip3 interacts with the HIRA proteins Hip1 and SIm9 and is required for transcriptional silencing and accurate chromosome segregation. J Biol Chem 281:8732-8739

Grewal SI (2000) Transcriptional silencing in fission yeast. J Cell Physiol 184:311-318

Haldar D, Kamakaka RT (2008) Schizosaccharomyces pombe Hst4 functions in DNA damage response by regulating histone $\mathrm{H} 3 \mathrm{~K} 56$ acetylation. Eukaryot Cell 7:800-813

Huang S, Zhou H, Tarara J, Zhang Z (2007) A novel role for histone chaperones CAF-1 and Rtt106p in heterochromatin silencing. EMBO J 26:2274-2283

Kaufman PD, Kobayashi R, Stillman B (1997) Ultraviolet radiation-sensitivity and reduction of telomeric silencing in Saccharomyces cerevisiae cells lacking Chromatin Assembly Factor-I. Genes Dev 11:345-357

Kunoh T, Habu T, Matsumoto T (2008) Involvement of fission yeast Clr6-HDAC in regulation of the checkpoint kinase Cds1. Nucleic Acids Res 36:3311-3319

Lewis LK, Karthikeyan G, Cassiano J, Resnick MA (2005) Reduction of nucleosome assembly during new DNA synthesis impairs both major pathways of double-strand break repair. Nucleic Acids Res 33:4928-4939

Li B, Carey M, Workman JL (2007) The role of chromatin during transcription. Cell 128:707-719

Linger J, Tyler JK (2005) The yeast histone chaperone chromatin assembly factor 1 protects against double-strand DNA-damaging agents. Genetics 171:1513-1522

Maas NL, Miller KM, DeFazio LG, Toczyski DP (2006) Cell cycle and checkpoint regulation of histone H3 K56 acetylation by Hst3 and Hst4. Mol Cell 23:109-119

Marheineke K, Krude T (1998) Nucleosome assembly activity and intracellular localization of human CAF-1 changes during the cell division cycle. J Biol Chem 273:15279-15286

McGowan CH, Russell P (2004) The DNA damage response: sensing and signaling. Curr Opin Cell Biol 16:629-633

McNeely S, Beckman R, Lin AB (2013) CHEK again: Revisiting the development of CHK1 inhibitors for cancer therapy. Pharmacol Ther 40:109-117

Mellone BG, Ball L, Suka N, Grunstein MG, Partidge JF, Allshire RC (2003) Centrosome Silencing and function in fission yeast is governed by the amino terminus of histone $\mathrm{H} 3$. Curr Biol 13:1748-1757

Moreno S, Klar A, Nurse P (1991) Molecular genetic analysis of fission yeast Schizosaccharomyces pombe. Methods Enzymol 194:795-823

Nabatiyan A, Szüts D, Krude T (2006) Induction of CAF-1 expression in response to DNA strand breaks in quiescent human cells. Mol Cell Biol 26:1839-1849

Nakayama J, Xiao G, Noma K, Malikzay A, Bjerling P, Ekwall K, Kobayashi R, Grewal SI (2003) Alp13, an MRG family protein, is a component of fission yeast Clr6 histone deacetylase required for genomic integrity. EMBO J 22:2776-2787

Ridgway P, Almouzni G (2000) CAF-1 and the inheritance of chromatin states: at the crossroads of DNA replication and repair. J Cell Sci 113:2647-2658

Rothstein RJ (1983) One-step gene disruption in yeast. Methods Enzymol 101:202-211

Saunders MS, Kalashnikova T, Smith MM (2006) Breaking barriers to transcription elongation. Nat Rev Mol Cell Biol 7:557-567

Sharp JA, Rizki G, Kaufman PD (2005) Regulation of histone deposition proteins Asf1/Hir1 by multiple DNA damage checkpoint kinases in Saccharomyces cerevisiae. Genetics 171:885-899
Shibahara K, Stillman B (1999) Replication-dependent marking of DNA by PCNA facilitates CAF-1-coupled inheritance of chromatin. Cell 96:575-585

Smith S, Stillman B (1989) Purification and characterization of CAFI, a human cell factor required for chromatin assembly during DNA replication in vitro. Cell 58:15-25

Taddei A, Roche D, Sibarita JB, Turner BM, Almouzni G (1999) Duplication and maintenance of heterochromatin domains. J Cell Biol 147:1153-1166

Takahashi K, Murakami S, Chikashige Y, Funabiki H, Niwa O, Yanagida M (1992) Alow copy number central sequence with strict symmetry and unusual chromatin structure in fission yeast centromere. Mol Biol Cell 3:819-835

Takami Y, Ono T, Fukagawa T, Shibahara K, Nakayama T (2007) Essential role of chromatin assembly factor-1-mediated rapid nucleosome assembly for DNA replication and cell division in vertebrate cells. Mol Biol Cell 18:129-141

Tamburini BA, Carson JJ, Linger JG, Tyler JK (2006) Dominant mutants of the Saccharomyces cerevisiae ASF1 histone chaperone bypass the need for CAF-1 in transcriptional silencing by altering histone and Sir protein recruitment. Genetics 173:599-610

Tanaka K, Boddy MN, Chen XB, McGowan CH, Russell P (2001) Threonine-11, phosphorylated by Rad3 and atm in vitro, is required for activation of fission yeast checkpoint kinase Cds1. Mol Cell Biol 21:3398-3404

Zhao H, Tanaka K, Nogochi E, Nogochi C, Russell P (2003) Replication checkpoint protein Mrc1 is required by Rad3 and Tel1 in fission yeast. Mol Cell Biol 23:8395-8403

doi:10.1186/2193-1801-3-30

Cite this article as: Kunoh and Habu: Pcf1, a large subunit of CAF-1, required for maintenance of checkpoint kinase Cds1 activity. SpringerPlus 2014 3:30.

\section{Submit your manuscript to a SpringerOpen ${ }^{\odot}$ journal and benefit from:}

- Convenient online submission

$\checkmark$ Rigorous peer review

- Immediate publication on acceptance

- Open access: articles freely available online

- High visibility within the field

- Retaining the copyright to your article

Submit your next manuscript at $>$ springeropen.com 